\title{
Cálculo Numérico do Espalhamento Eletromagnético por Cilindros Inomogêneos
}

\author{
Carlos Gustavo S. Migliora e Miriam B. Fernandes Chaves
}

O espalhamento de ondas eletromagnéticas por cilindros dielétricos inomogêneos, de seçäo reta arbitrắria, es tratado por uma formulaçăo baseada no método do unimomento. - problema interior de elementos finitos es resolvido sobre uma malha cuja geometria aumenta a eficiência computacional em relação àquelas comumente utilizadas. Resultados assim obtidos săo apresentados e comparados com outros publicados na literatura e provenlentes de métodos diversos.

\section{Introđução}

A determinação do diagrama de espalhamento para corpos iluminados por ondas eletromagnéticas constitui um importante problema na área de eletromagnetismo aplicado com aplicação, por exemplo, no cálculo do espalhamento por gotas de chuva, tecidos biológicos e em prospecção geofísica. As aproximaçōes assintóticas, usualmete utilizadas para baixas freqüências (Rayleigh e Born) ou altas freqüências (WKB, "Wentzel-Kramers-Brillouin" e GTD, "Geometrical Theory of Diffraction"), falham na chamada região de ressonância, onde as dimensōes envolvidas são da ordem de grandeza dos comprimentos de onda utilizados. Torna-se aí necessária uma soluçāo precisa para as equaçōes de Maxwell.

No caso do espalhamento por cilindros dielétricos infinitos, dispōe-se de soluçōes quando estes são hornogêneos e de seção reta circular ou elítica. Para outras formas de seção reta, ou quando ocorrem inomogeneidades, apenas aproximações são conhecidas.

Os problemas de espalhamento têm sido frequentement? formulados através de equaçŏes integrais [1] e [2], em detrimento de abordagens diferenciais, pois a regiāo de solução da equação integral se restringe à superfície do corpo e a condição de radiação é automaticamente considerada. Este tipo de formulação, entretanto, envolve problemas extremamente difíceis quando

C.G.S. Migilora Professor do Centro de Estudos em Telecomunicaçס̋es da PUC/Rio, 22453, Rilo de Janeiro, RJ.

M.B.F. Chaves é Assistente de Pesquisa no Laboratório Nacional de Computação Cientifica - CNPq, Caixa Postal 56018, 22290, Rio de Janeiro, RJ. 
os meios tratados são inomogêneos ou anisotrópicos [3] e [4]. Por sua vez, uma formulação diferencial usando elementos finitos ou diferenças finitas é conveniente para meios inomogênèos, mas a condição de radiação no infinito não pode ser imposta de forma simples. Além disso, a região considerada nas soluçōes numéricas deve envolver todo o corpo, o que gera matrizes de dimensões muito grandes para serem manipuladas, mesmo em computadores modernos de grande porte. Para contornar estas dificuldades, diversos procedimentos têm sido propostos por vários autores [5] - [9] .

O objetivo deste trabalho é apresentar uma solução para os problemas de espalhamento eletromagnético por cilindros dielétricos infinitos, inomogêneos e de seção reta arbitrária, porém uniforme. A formulação desenvolvida na Seção 2 segue o método do unimomento, introduzido por Mei [10] em 1974.

Assim, os campos nas regiōes exterior, de fronteira livre, e interior, de contorno fechado, são expressos, respectivamente, por uma expansão modal e por um conjunto de funções teste geradas numericamente. A igualdade dos campos é, então, forçada (por mínimos quadrados) sobre um círculo artificial que envolve completamente a regiāo inomogênea, fornecendo os coeficientes (incógnitas) da expansão exterior.

A grande vantagem desta técnica reside em sua simplicidade e eficiência permitindo desacoplar efetivamente o problema interior de vaiores de contorno, do problema exterior de espalhamento. Em particular, quando o método de elementos finitos (MEF) é empregado, como descrito na Seção 3, as matrizes resultantes são blocadas e esparsas, o que é muito conveniente do ponto de vista das técnicas de arnazenamento e inversão normalmente utilizadas. A geometria da malha de elementos finitos está intimamente relacionada com a complexidade e o tempo de computação necessários para a solução. Uma malha regular com elementos triangulares de tamanho fixo [11] é muito simples de gerar, mas tem o inconveniente de exigir um cuidado especial com as possíveis mudanças abruptas do material nos elementos próximos ao contorno do corpo. Um tratamento unificado para este tipo de malha é, portanto, impossivel, o que aumenta as dificuldades de implementação.

Uma geometria mais conveniente, como a das chamadas malhas conformes [12] $€[13]$, garante que a fronteira (arbitrária) do cilindro coincida com interfaces de elememtos. Usando, ainda, uma densidade suficienternente grande que permita desprezar pequenas variaçōes do material dentro de cada elemento. um único procedimento pode ser aplicado em toda a malha, reduzindo assim o esforço computacional.

A implementação da metodologia desenvolvida nas seções 2 e 3 é apresentada, a seguir, na Seção 4, através da descrição do programa ESPEMC 
(Espalhamento Eletromagnético por Cilindros), cujos módulos principais solução do problema interior de elementos finitos (EF) e acoplamento das soluções - são, ali, comentados. Finalmente, na Seção 5 , são fornecidos vários resultados obtidos com o programa, comprovando sua precisão e convergência numérica.

\section{O Problema Bidimensional de Espalhamento}

O problema de interesse consiste em obter o campo espalhado pelo cilindro dielétrico infinito, uniforme na direção $z$, mostrado na Fig. 1. A seção reta $C$ do cilindro é arbitrária e o dielétrico tem permissividade relativa $\varepsilon(r)$ variando continuamente ou discretamente com a posição definida pelo vetor r. O campo incidente tem como única restrição a direção de incidência normal a $z$, podendo corresponder a uma onda plana, a uma onda cilíndrica gerada por uma linha de corrente ou a qualquer outra função conhecida [11] . Assume-se, ainda, a convencional dependência harmônica com o tempo da forma exp ( $j \omega t)$. Como todas as quantidades são invariantes com relação a $z$, os campos eletromagnéticos totais podem ser obtidos diretamente das componentes axiais do campo elétrico $\left(E_{z}\right)$, no caso de ondas transversais magnéticas $\left(T M_{Z}\right)$, ou do campo magnético $\left(H_{Z}\right)$, para ondas transversais elétricas $\left(T E_{Z}\right)$ [14].

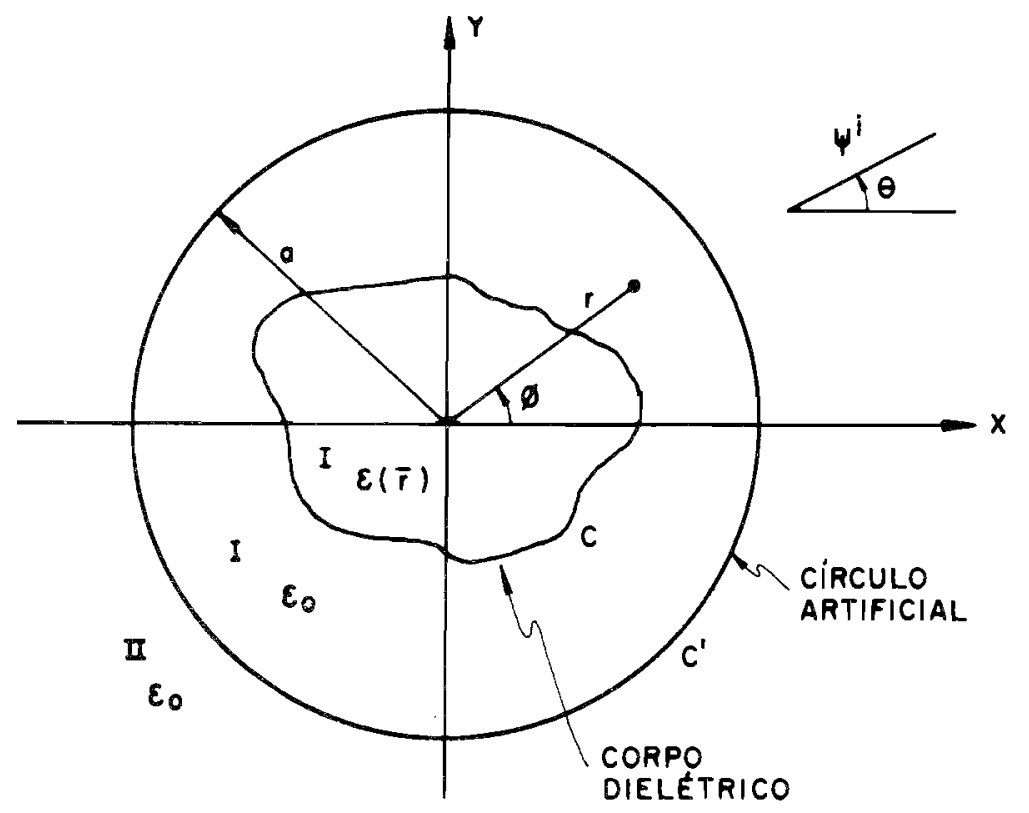

Figura 1. Cilindro dielétrico e círculo artificial. 
O método do unimomento aplicado a este problema consiste em traçar o círculo artificial C' de raio a (ver Fig.1), de modo a envolver completamente a regiāo inomogênea e resolver, em separado, os problemas interior $(r<a$, regiấo I) e exterior ( $r>a$, região II), como mostra a Fig. 1. As condiçōes de contorno para os campos $E$ e $H$ são agora consideradas em $C^{\prime}$ e não na fronteira $C$ do cilindro. Em outras palavras, elas passam a ser simples condiçōes de continuidade num contorno bem comportado (fronteira separável).

Na regiáo II, o campo total é a soma do campo incidente com o espalhado, ou seja

$$
\psi^{\prime \prime}(\mathbf{r})=\psi^{i}(\mathbf{r})+\psi^{\mathbf{e}}(\mathbf{r})
$$

onde $\mathbf{r}=(r, \phi)$ e

$$
\psi(\mathbf{r})=\left\{\begin{array}{l}
E_{Z}(\mathbf{r}), \text { para ondas } T M_{z^{\prime}} \\
H_{z}(\mathbf{r}), \text { para ondas } T E_{Z^{\prime}}
\end{array}\right.
$$

O campo incidente $\psi^{i}$ é uma função conhecida e o campo espalhado $\psi^{e}$ é expresso por uma expansão em harmônicos cilíndricos da forma

$\psi^{e}(\mathbf{r})=\sum_{n=0}^{N} B_{n} H_{n}{ }^{(2)}\left(k_{o} r\right) e^{j n \phi}=\sum_{n=0}^{2 N} C_{n} T_{n}(\mathbf{r})$,

sendo

$$
T_{n}(\mathbf{r})=\left\{\begin{array}{l}
H_{n}^{(2)}\left(k_{0} r\right) \cos (n \phi), n \leqslant N, \\
H_{n}^{(2)}\left(k_{0} r\right) \operatorname{sen}[(n-N) \phi], n>N,
\end{array}\right.
$$

onde $H_{n}{ }^{(2)}$ é a função de Hankel de segunda espécie e ordem $n$ e $k_{0}$ $=\omega\left(\mu_{0}^{n} \epsilon_{0}\right) 1 / 2$, com $\mu_{0}$ e $\epsilon_{0}$ representando a permeabilidade magnética e a permissividade elétrica do vácuo, respectivamente. A validade desta representação é garantida pela hipótese de Rayleigh [15]. O número de termos necessários para uma boa representação do campo espalhado, segundo a análise das referências [16] e [17], deve ser $2 \mathrm{~N}+1>\mathrm{ka}$, onde a é o raio do círculo $C^{\prime}$. 
Na região I, o campo será representado por uma combinação linear de funções teste $\psi_{n}(\mathbf{r})$ que devem satisfazer à equação de Helmholtz bidimensional

ou

$$
\left(\nabla^{2}+k_{0}^{2} \epsilon_{r}(\mathbf{r})\right) \psi_{n}(\mathbf{r})=0 \text {, para ondas } \mathrm{TM}_{\mathrm{z}}
$$

$$
\left(\nabla \cdot\left(\epsilon_{\vec{r}}^{-1}(\boldsymbol{r})\right)+k_{0}^{2}\right) \psi_{n}(\mathbf{r})=0 \text {, para ondas } T E_{z^{\prime}}
$$

tendo como condiçóes de contorno, ao longo de $\mathrm{C}^{\prime}$, o campo incidente ou os modos da expansão exterior, ou seja

$\psi_{n}(a, \phi)= \begin{cases}T_{n}(a, \phi), n=0, \ldots, 2 N, & \\ \psi^{i}(a, \phi), n=2 N+1 . & 0<\phi \leqslant 2 \pi\end{cases}$

Denotando por $\widehat{T}_{n}(\boldsymbol{r}), n=0, \ldots, 2 \mathrm{~N}$ e por $\hat{\psi}_{i}(\mathbf{r})$ as funções teste resultantes das condições de contorno correspondentes, o campo na regiảo I pode ser escrito na forma

$$
\widehat{\psi^{\prime}}(\mathbf{r})=\hat{\psi^{i}}(\mathbf{r})+{\underset{n=0}{2 N}}_{n} c_{n} \widehat{\hat{T}}_{n}(r)
$$

Estas funções são geradas por elementos finitos, conforme a discussão na Seção 3.

Estabelecidas as representaçōes para os campos nas regiões I e II, o próximo passo é satisfazer à condição de continuidade sobre o contorno $\mathrm{C}^{\prime}, \mathrm{O}$ que será discutido a seguir.

\section{Acoplamento das Soluçóes}

A determinação das funções que representam o campo na região interna ao circulo C' pelo método de elementos finitos, segundo a discussão na Seção 3, requer a construção de uma malha como a apresentada na Fig. 2 , sendo os valores de $\psi^{\prime}(\mathbf{r})$ definidos sobre os nós desta malha. Observando-se que as condiçōes de contorno em (5) já fixam valores deste campo em $\mathrm{r}=\mathrm{a}$, isto é, sobre os nós do círculo $\mathrm{C}^{\prime}$, as condições de continuidade devem ser impostas em outro círculo, por exemplo, no círculo seguinte da malha ( $r=r_{1}$, na Fig. 2), ou seja

$\widehat{\psi^{i}}\left(r_{1}\right)+\sum_{n=0}^{2 N} C_{n} \widehat{T}_{n}\left(r_{1}\right)=\widehat{\psi^{i}}\left(r_{1}\right)+\sum_{n=0}^{2 N} C_{n} T_{n}\left(r_{1}\right)$. 


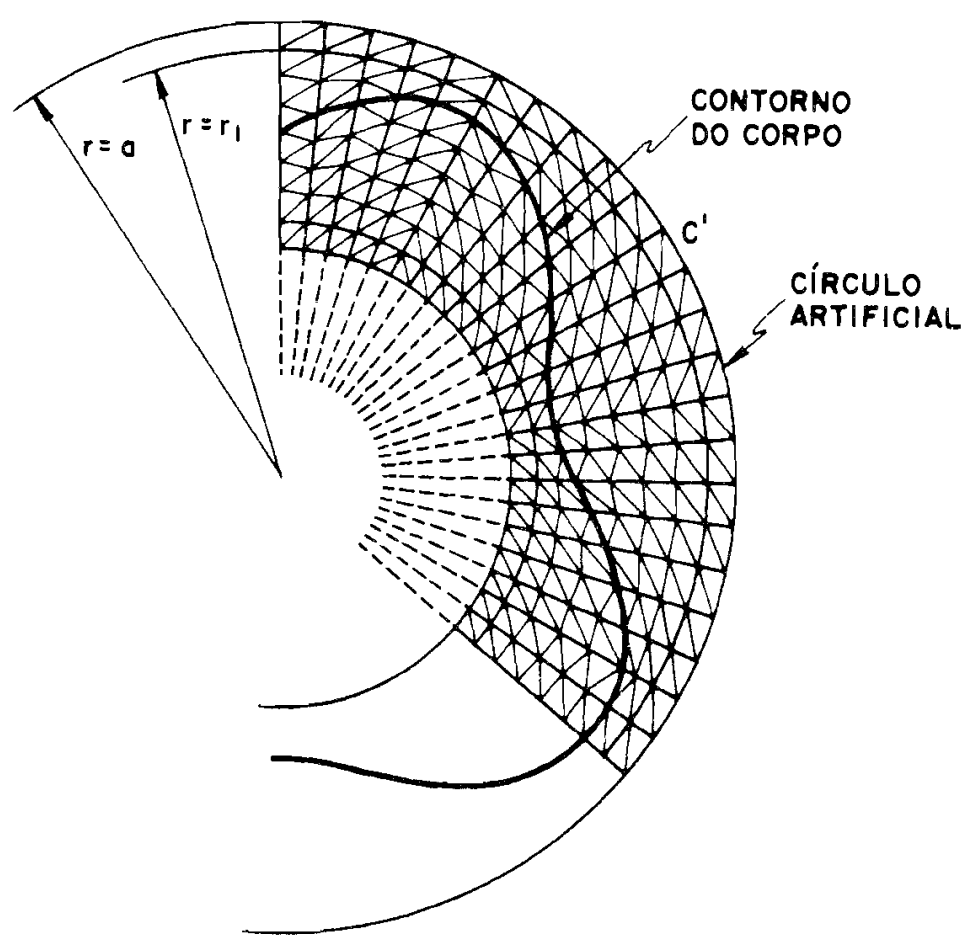

Figura 2. Malha anular conforme de elementos finitos.

to corresponde a estender a representação do campo na região II um suco para dentro da região $\mathrm{I}$, mais precisamente até o raio $r=r_{1}$.

sta igualdade seria exata caso a expansão em (2) não fosse truncada se fosse possível resolver com total precisão cada problema interior definido n (4) e (5). Como, na realidade, as somas são truncadas e as funções roximadas, a determinação dos coeficientes se faz de modo a forçar a jaldade dos campos no sentido dos mínimos quadrados. O critério para terminação dos coeficientes complexos $C_{n}$ consiste em requerer a menor lerença entre as duas representações em (7).

: finindo os campos de erro incidente e modal, em $r=r_{1}$, respectivamente $r$

$$
\begin{aligned}
& d \psi^{i}\left(r_{1}\right)=\widehat{\psi}^{i}\left(r_{1}\right)-\psi^{i}\left(r_{1}\right) \\
& d T_{n}\left(r_{1}\right)=\widehat{T}_{n}\left(r_{1}\right)-T_{n}\left(r_{1}\right),
\end{aligned}
$$


é possivel escrever uma expressão para o campo de erro total, da seguinte forma

$$
\begin{aligned}
& \mathrm{d} \psi^{\mathrm{T}}\left(r_{1}\right)=\psi^{\prime}\left(r_{1}\right)-\psi^{\prime \prime}\left(r_{1}\right) \\
& =\mathrm{d} \psi^{i}\left(r_{1}\right)+\sum_{n=0}^{2 N} \mathrm{C}_{\mathrm{n}} \mathrm{dT} \mathrm{n}_{n}\left(r_{1}\right) .
\end{aligned}
$$

Usando a norma Euclideana para expressar o erro médio quadrático, $e^{2}$ tem-se

$$
e^{2}=\left\|d \psi^{t}(\mathbf{r})\right\|_{r_{1}}^{2}
$$

onde

$$
\|f\|_{r}^{2}=(f, f)_{r}=\int_{0}^{2 \pi} f(r, \phi) f^{*}(r, \phi) d \phi
$$

Aplicando (9) em(10), após alguma manipulação algébrica chegamos à expressāo do erro quadrático médio

$$
\begin{aligned}
e^{2}=\left\|d \psi^{i}\right\|_{r_{1}}^{2} & +2 R e\left\{\sum_{n=0}^{2 N} C_{n}\left(d T_{n^{\prime}} d \psi^{i}\right)_{r_{1}}\right\} \cdots+ \\
& +\sum_{n=0}^{2 N} \sum_{\ell=0}^{2 N} C_{n} C_{\ell}^{*}\left(d T_{n^{\prime}} d T_{\ell}\right)_{r_{1}}
\end{aligned}
$$

onde $\operatorname{Re} \quad\{$.$\} representa a parte real da quantidade entre chaves e (., .)_{r}$ denota o produto interno conjugado induzido pela norma em(11).

Para minimizar este erro usa-se a condição de derivada nula com respeito a cada incógnita $C_{n}$. As partes real e imaginária são separadas e denotadas por $w_{n}=\operatorname{Re}\left\{C_{n}\right\}$ e $y_{n}=\operatorname{Im}\left\{C_{n}\right\}$ e a condição de nulidade, uma vez estabelecida, fornece o sistema linear

$$
\begin{aligned}
\frac{\partial e^{2}}{\partial w_{k}}+j \frac{\partial e^{2}}{\partial y_{k}} & =\sum_{n=0}^{2 N} G_{n}\left(d T_{n^{\prime}}, d T_{k}\right)_{r_{1}}+\left(d \psi^{i}, d T_{k}\right)_{r_{1}}=0, \\
k & =0, \ldots, 2 N,
\end{aligned}
$$


cuja solução gera os coeficientes desconhecidos $C_{n}$. A matriz de acoplamento é formada pelos produtos internos dos campos de erro modais (dT $n, d T_{k}$ ), $n, k_{1}=0, \ldots, 2 \mathrm{~N}$, e o vetor de acoplamento é constituído pelos produtos internos do campo de erro incidente por cada um dos campos de erro modais $\left(\mathbf{d} \psi^{i}, \mathrm{dT} k\right), \mathrm{n}, \mathrm{k}=0, \ldots, 2 \mathrm{~N}$. Fica claro, portanto, que uma vez obtida a matriz de acoplamento, resolver o sistema para novos campos incidentes representa apenas um pequeno acréscimo no esforço computacional. Esta é uma das grandes vantagens do unimomento, pois isso permite a solução simultânea para vários campos incidentes.

\section{Solução Numérica do Problema Interior}

O método do unimomento presume a habilidade de resolver numericamente, de forma precisa, cada problema interior de valores de fronteira formulado em (4) e (5), gerando o conjunto de funçōes teste usadas para representar - campo na regiáo I da Fig. 1. A aplicação do MEF a esses problemas baseia-se na representação variacional das equações de Helmholtz [18]. Pelo princípio estacionário [18], as soluçōes destas equaçōes sāo obtidas determinando-se as funçōes que tornam estacionários os funcionais

$$
\begin{aligned}
& F=\int_{S}\left\langle\left|\nabla \psi_{n}\right|^{2}-k_{o}^{2} \epsilon_{r} \psi_{n}^{2}\right\rangle d s \text {, para ondas } T M_{z} \\
& \quad F=\int_{S}\left\{\epsilon_{r}^{-1}\left|\Delta \psi_{n}\right|^{2}-k_{o}^{2} \psi_{n}^{2}\right\rangle d s \text {, para ondas } T E_{z}
\end{aligned}
$$

onde $S$ é a área limitada pelo círculo $C^{\prime}$ e as condições de contorno são do tipo Dirichlet, como em (5).

O primeiro passo na soluçäo de EF consiste em decompor a regiáo interior em elementos triangulares. Os campos dos funcionais em (14) são expandidos em funções de base piramidais $u_{n m}(x, y)$ da seguinte forma

$$
\psi_{n}(x, y)=\sum_{m=1}^{M} a_{n m} u_{n m}(x, y)
$$

onde $M$ é o número total de nós. $O$ conjunto $a_{n m}, m=1, \ldots M$, representa os valores de $\psi_{n}(x, y)$, desconhecidos para os $M_{i}$ nós internos mas conhecidos para os $M_{c}$ nós da fronteira $\left(M=M_{c}+M_{i}\right)$. $O$ índice $n$, relacionado à representação do campo na região interior, será omitido daqui para frente para facilitar a notação. 
Aplicando os procedimentos usuais de EF [19], a determinação do ponto estacionário do funcional (14a) resulta no sistema linear expresso por

$$
\begin{gathered}
\sum_{j=1}^{G(m)} \sum_{k=1}^{3}\left\{\left(U_{\ell}^{r}\right)^{t} \cdot v_{\ell} \cdot U_{\ell}^{k}-k_{o}^{2} \epsilon_{r}(\ell) \cdot\left(U_{\ell}^{r}\right)^{t} \cdot W_{\ell} \cdot U_{\ell}^{k} \cdot q_{m}=0,\right. \\
m=1, \ldots, M ;
\end{gathered}
$$

onde $m=v(l, r)$ é $o$ índice dos nós do elemento $l=\mu(m, j), \quad \nu$ e $\mu$ são as matrizes de conexão entre nós e elementos [20] e $G(m)$ fornece o número de elementos agrupados em torno do nó $\mathrm{m}$. O vetor $\cup_{\ell}^{r}$ é formado pela coluna $r$ da matriz $U_{\ell}$ que descreve funções de base sobre o elemento e

$\mathbf{U}_{\ell}=\frac{1}{2 A_{\ell}}\left[\begin{array}{ccc}y_{2}-y_{3} & y_{3}-y_{1} & y_{1}-y_{2} \\ x_{3}-x_{2} & x_{1}-x_{3} & x_{2}-x_{1} \\ x_{2} y_{3}-x_{3} y_{2} & x_{2} y_{1}-x_{1} y_{3} & x_{1} y_{2}-x_{2} y_{1}\end{array}\right]$

sendo $A_{\ell}$ a área do elemento triangular cujos vértices têm coordenadas $\left(x_{i}, y_{i}\right), \quad i=1,2,3$. As matrizes elementares de integração $v_{\ell}$ e $\mathbf{w}_{\ell}$ têm a forma

$$
v_{\ell}=\int_{S_{\ell}}\left[\begin{array}{lll}
1 & 0 & 0 \\
0 & 1 & 0 \\
0 & 0 & 0
\end{array}\right] d x d y
$$

$$
W_{\ell}=\int_{S_{\ell}}\left[\begin{array}{lll}
x^{2} & x y & x \\
x y & y^{2} & y \\
x & y & 1
\end{array}\right] \quad d x d y \text {, }
$$

onde $S_{2}$ denota a regiâo do elemento $\ell$ e as integrais são do tipo

$$
P_{r s}=\int_{S_{\ell}} x^{r} y^{s} d x d y,
$$

cujas expressões são conhecidas [21] . 
A equação global do sistema, cuja solução fornece os valores nodais internos do campo, em termos de seus valores no contorno, pode ser obtida diretamente de [16], uma vez especificada a topologia e a numeração de malha através das matrizes de conexão nó-elemento [20]. A seguir será descrita a malha anular conforme usada nesta solução do problema de espalhamento.

\section{A Malha Anular Conforme}

Dentre as malhas usualmente utilizadas, a mais simples é a que mantém fixa a geometria dos elementos, sendo construida independentemente do contorno do corpo. Isto implica na ocorrência de elementos em cujo interior o material pode variar abruptamente, sobretudo na fronteira do cilindro. Assim, a integração vai depender de o elemento apresentar ou não essa descontinuidade, o que aumenta a complexidade da implementação e torna mais lento o cálculo dos campos.

Para eliminar este problema, será usada a malha anular conforme mostrada na Fig. 2, cuja principal característica é a coincidência do contorno do corpo com interfaces entre elementos. Com uma densidade suficientemente grande que permita desprezar a variação do material dentro de cada triângulo - hipótese de elementos homogêneos - garante-se um procedimento computacional unificado que não depende da malha.

A construção da malha anular conforme se faz sequencialmente, por intervalos angulares constantes, começando do eixo vertical e prosseguindo no sentido horário. A cada passo, a malha é ajustada de forma que a aproximação do contorno do corpo por segmentos de reta coincida com as interfaces dos elementos, minimizando as distorções [20]. A Fig. 3 mostra a topologia e a numeração dos nós da malha, nas vizinhanças do i-ésimo intervalo angular.

A numeração global se baseia na estrutura da malha que pode ser relacionada com uma matriz, isto é, a cada nó está associado um índice $m=(j-1)$. $J+i$, onde $i=1, \ldots, \mid$ é o número da linha radial definida pelo ângulo $\phi_{i}$ e $j=1, \ldots, J$ é o número dos nós sobre cada uma destas linhas.

O sistema matriciai resultante da implementação do MEF tem como principal característica uma estrutura blocada de submatrizes. Observa-se da Fig. 3 que cada nó da linha i está ligado apenas a nós das linhas adjacentes $i-1$ e $i+1$. Definindo o vetor de incógnitas nodais da i-ésima linha radial por $q_{i}=[q(i, 1), \ldots, q(i, J)] e$ usando o sistema expresso em $[16]$, obtém-se o sistema local

$$
a_{i} q_{i-1}+b_{i} q_{i}+c_{i} q_{i}+1=P_{i}, i=1, \ldots, i
$$




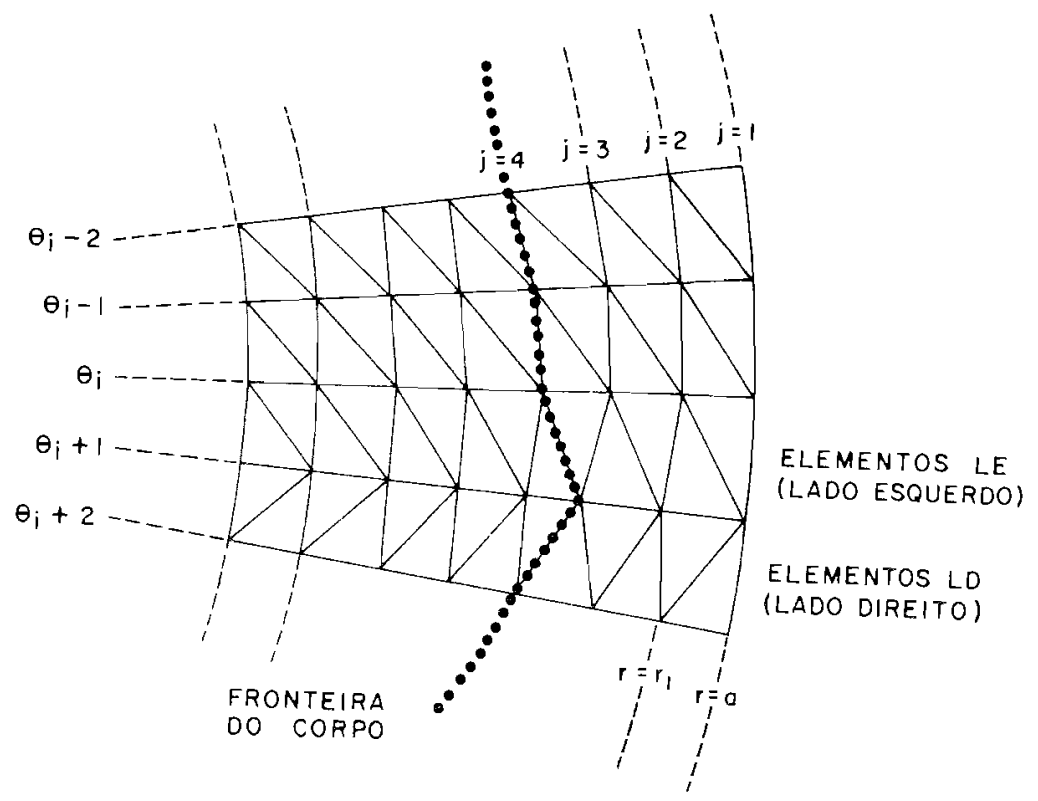

Figura 3. Construção local da malha anular conforme.

onde $\mathbf{a}_{\mathfrak{i}}, \mathbf{b}_{\mathbf{j}}$ e $\mathbf{c}_{\mathbf{i}}$ são matrizes de banda, $\mathrm{J} \times \mathrm{J}$, com as estruturas mostradas na Fig. 4. Observa-se a dependência de ai e ci com a orientação (LD, lado direito ou LE, lado esquerdo) dos elementos entre as linhas $\mathrm{i}-1 \mathrm{e} i+1$.

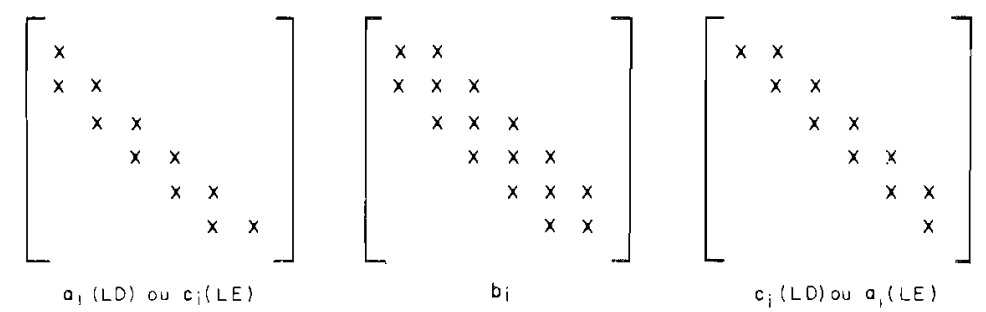

Figura 4. Estrutura dos blocos $a_{\mathbf{i}}, b_{\mathbf{j}} \in \mathbf{c}_{\mathfrak{j}}$.

A úrica componente não nula do vetor $\mathrm{P}_{\mathrm{i}}$ é a primeira, correspondendo ao nó prescrito do contorno.

A matriz global $\mathbf{T}$ do sistema é construída usando as submatrizes $\mathbf{a}_{\mathbf{i}}, \mathbf{b}_{\mathbf{j}}$ $\in \mathbf{c}_{i}$, como ilustra a Fig. 5, e sua estrutura resulta da ordenação dos nós 
na mesma seqüência em nue eles aparecem na malha Os blocos aj e $\mathbf{c}_{\mathbf{j}}$ aparecem fora da banda porque representam as contribuiçōes dos elementos do último setor no primeiro e vice-versa. Também o nó central possui características distintas, pois recebe contribuições de um elemento de cada setor.

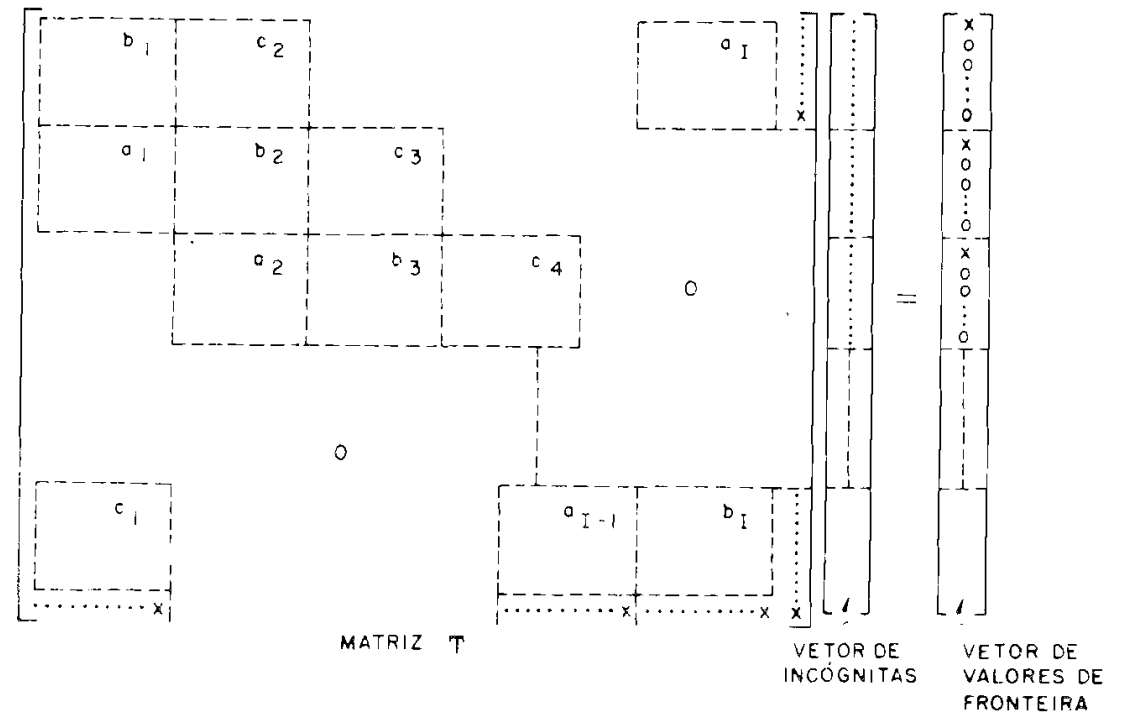

Figura 5. Estrutura da matriz global de elementos finitos.

\section{Implementação}

Nas seções anteriores, discutiu-se a aplicação do método do unimomento em conjunto com elementos finitos ao cálculo do espalhamento eletromagnético por cilindros dielétricos inomogêneos. Aspectos computacionais relativos à implementação desta metodologia no desenvolvimento do programa ESPEMC, escrito em FORTRAN IV e projetado de forma modular, serão comentados a seguir.

O primeiro conjunto de ações que o programa executa consiste na leitura dos dados de entrada, ou seja:

a) freqüência dos campos envolvidos;

b) nivel de refinamento da malha anular conforme, isto é, o número de intervalos angulares e radiais;

c) características dos campos incidentes que serão analisados pelo programa FSPEMC;

12 
d) o número de termos usados nas expansões que representam os campos, nas regiões interior e exterior;

e) permissividade relativa $\epsilon_{\mathrm{r}}(\mathbf{r})$.

O módulo 2, a seguir, realiza os processos do MEF que permitem obter as funçōes $\hat{\psi}$ ie $\widehat{T}_{n}$. Isto inclui a geração automática da malha anular conforme, a especificação das matrizes de conexão nó-elemento $(\nu$ e $\mu$ ), a construção da matriz global, a imposição das condições de continuidade $e$ a resolução do sistema linear de elementos finitos.

No módulo 3 são calculados os termos da matriz e do vetor de acoplamento, resolvendo-se, em seguida, o sistema linear complexo que fornece os coeficientes $C_{n}$ das expansōes que representam os campos nas regiōes interior e exterior. Caso haja mais de um campo incidente, um conjunto de coeficientes é gerado para cada campo.

O último módulo (módulo 4) destina-se à geração de relatórios e arquivos de saída que descrevem as características do experimento realizado e listam: os coeficientes $C_{n}$ calculados. Rotinas externas produzem os gráficos do diagrama de espalhamento ou da largura de eco obtidos a partir destes coeficientes.

\subsection{Módulo 2: Algoritimo de Elementos Finitos}

Diversas formas de implementação do MEF têm sido apresentadas e discultidas na literatura. Estas abordagens guardam em comum uma grande generalidade, de forma a permitir a resolução de problemas envolvendo geometrias diversas e condições de contorno complexas. Na realização do móduı 2 (geração das funções $\hat{\psi}_{e} \widehat{T}_{n}$ ) foram utilizadas algumas rotinas de uso mais geral, adaptadas de [22], e projetadas outras específicas, respeitardo as características do problerna de espalhamento.

A eficiência de um programa de elementos finitos depende, entre outros fatores, da escolha apropriada da forma de armazenagem das matrizes e das técnicas numéricas empregadas para resolıx r o problema linea: resultante. Considerando a estrutura particular, simétrica e esparsa, da matriz global do sistema, foi escolhido um esquema de armazenamento do tipo "skyline", descrito detalhadamente em [23]. A característica mais importante desta técnica é a de armazenar apenas os elementos localizados entrı o primeiro termo não nulo de cada coluna e a diagonal principal. Com isso, o número de termos guardados é bem menor, permitindo o tratamento de malhas com número relativamente grande de pontos nodais. 
Quanto à solução do sistema linear, o algoritmo escolhido foi a eliminação de Gauss, implementado pela rotina COLSOL adaptada de [22].

Outro ponto crucial para o método de elementos finitos é a escolha de como tratar as condições de contorno. No problema de valores de fronteira definido por (4a) ou (4b), essas condições são expressas pelos valores das funções $\hat{\psi}^{i} \mathrm{e} \widehat{T}_{n}$ nos pontos nodais localizados sobre o círculo $C^{\prime}$ da Fig. 1. Optou-se, no caso, por montar a matriz global com as equações relativas a todos os nós, inclusive os do contorno $C^{\prime}$, e desenvolver uma rotina especificando os nós prescritos e modificando, convenientemente, a matriz.

\subsection{Módulo 3: Acoplamento das Soluçōes}

O módulo 3 foi desenvolvido com a finalidade de construir e resolver 0 sistema de acoplamento em (13), resultante do processo de minimização do erro entre as expansões usadas para representar os campos interior e exterior. Isso equivale a impor a condição de continuidade descrita por (7).

Em primeiro lugar, é preciso construir a matriz complexa formada pelos produtos internos $\left(d T_{n}, d T_{k}\right), n, k=0, \ldots, 2 N$, e os elementos $\left(d_{\psi} \psi^{i}, d T_{k}\right)_{r_{1}}$ $k=0, \ldots, 2 \mathrm{~N}$, do vetor de acoplamento que corresponde a cada campo incidente a ser considerado. Estes produtos internos envolvem integrais de contorno sobre os $\operatorname{arcos} \phi_{\mathbf{i}-1} \leqslant \phi \leqslant \phi_{\mathbf{j}+1}$ do círculo de raio $\mathrm{r}_{1}$, contidos nos elementos mais externos de cada setor $i$. Sendo assim, a primeira tarefa do módulo é identificar esses elementos, determinando a orientação (LD ou LE, ver Seção 3) dos triângulos no setor $i$.

Conhecidos os intervalos de integração e a numeração dos elementos, o próximo passo é calcular as integrais, determinando, assim, os termos do sistema de acoplamento.

Os coeficientes dos polinômios usados para expressar a solução por elementos finitos săo obtidos através da subrotina ELEM e do vetor de valores nodais das funçōes $\widehat{\psi}^{\mathrm{i}}$ e $\widehat{T}_{n}$ sobre o círculo de raio $r$. Sempre que possível, foram desenvolvidas rotinas com soluções exatas para as integrais definidas a serem calculadas. Apenas no caso do cálculo do vetor de acoplamento correspondente à onda plana foi preciso empregar a subrotina de integração numérica DCADRE do pacote matemático IMSL [24].

A última etapa do módulo 3 consiste em triangularizar a matriz complexa, usando-se para isso a subrotina LECT1C, também do IMSL [24]. Para cada 
campo incidente a considerar, constrói-se um vetor de acoplamento que, juntamente com a matriz triangularizada, gera, através de LECT1C, o coeficientes $C_{n}$ empregado na representação do campo espalhado pelo cilindro.

\section{Resultados e Conclusões}

O programa ESPEMC, comentado na seção anterior, foi aplicado ao cálculo do espalhamento por cilindros dielétricos com diversos tipos de seção reta e inomogeneidades. Com o intuito de comparar os resultados obtidos pelo programa com outras abordagens encontradas na literatura, foram calculados em todos os casos o diagrama de espalhamento $\mathrm{g}(\phi)$ ou a largura de eco $W(\phi)$, definidos por

$g(\phi)=C_{0}+\sum_{n=1}^{N} j^{n}\left[C_{n} \cos (n \phi)+C_{n+N} \operatorname{sen}(n \phi)\right]$

e

$W(\phi)=\left.\frac{2}{\pi} \lg (\phi)\right|^{2}$

Os parâmetros e caracteristicas comuns a todos os casos testados e apresentados nesta seção são:

i) onda plana incidente do modo $\operatorname{TM}_{z}\left(\psi=E_{z}\right)$;

ii) freqüência de $300 \mathrm{MHz}$;

iii) $\epsilon_{\mathrm{r}}=2$ ou 4;

iv) $0,4 \lambda \leqslant d_{\max }<1,0 \lambda$, onde $d_{\max }$ é a maior dimensão do corpo;

v) $0,25 \lambda<a<0,71 \lambda$;

vi) ângulos de incidência $\theta=0^{\circ}, 45^{\circ}$ ou $90^{\circ}$.

Estes valores foram propositadamente escolhidos de forma a permitir comparaçōes com resultados disponíveis.

O primeiro caso analisado foi o de um cilindro circular homogêneo de raio $r_{c}=0,3 \lambda$ e $\epsilon_{r}=4$. Para quebrar a simetria do problema, considerou-se o centro do círculo $C^{\prime}$, que define a malha de elementos finitos, deslocado de $D=0,1 \lambda$ do eixo do cilindro. Com isto, garante-se que a solução numérica não seja simplesmente uma integração da equação de Bessel como o seria se houvesse simetria angular na geometria. Como as funções geradas numeri- 
camente não dependem da onda incidente, a coincidência entre os centros da malha de elementos finitos e do cilindro circular homogêneo conduz a um problema unidimensional na coordenada radial, independente do ângulo $\phi$. A Fig. 6 mostra a malha anular conforme construida para este caso, e o diagrama de espalhamento $g(\phi)$ está na Fig. 7. Observa-se excelente concordância do resultado do programa com a solução exata obtida por separação de variáveis, com diferenças menores que $2 \%$ na curva de magnitude.

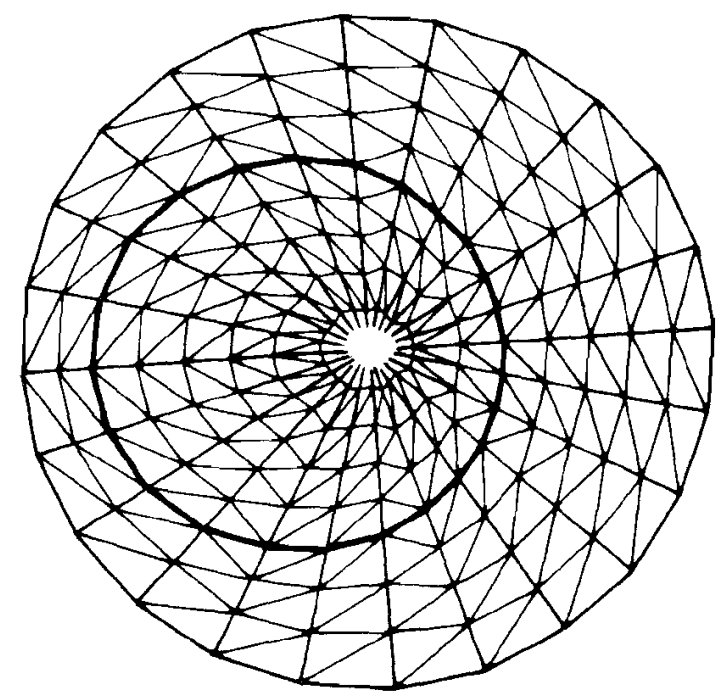

Figura 6. Malha anular conforme para o cilindro circular homogêneo com $\epsilon_{\mathrm{r}}=4$ e $\mathrm{r}_{\mathrm{c}}=0,3 \lambda$.

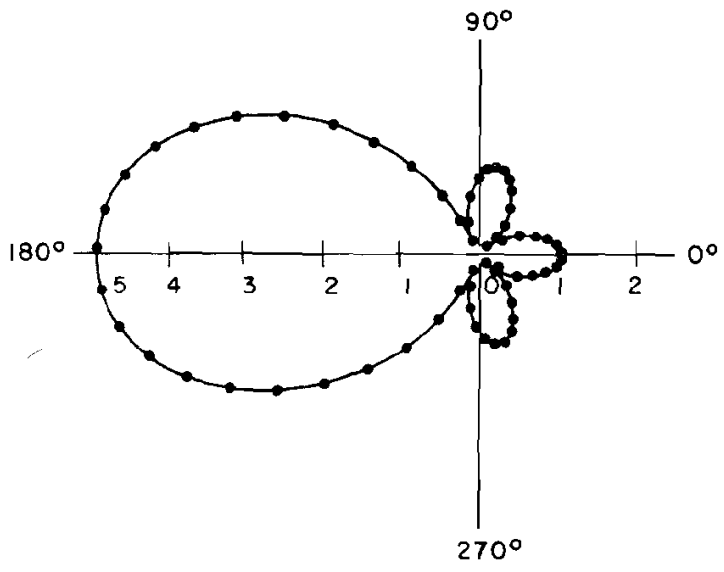

Figura 7. Diagrama de espalhamento do cilindro com $\epsilon_{r}=4$ e $r_{c}=0,3 \lambda$. 
Em seguida, foram calculados os diagramas de espalhamento para um cilindro elítico e para dois cilindros circulares superpostos. Nas figuras 8 e 9 estão mostradas as magnitudes de $\mathrm{g}(\phi)$ obtidas e as geometrias correspondentes, bem como os resultados contidos em [11] e [25], respectivamente. As diferenças observadas são menores que $10 \%$.

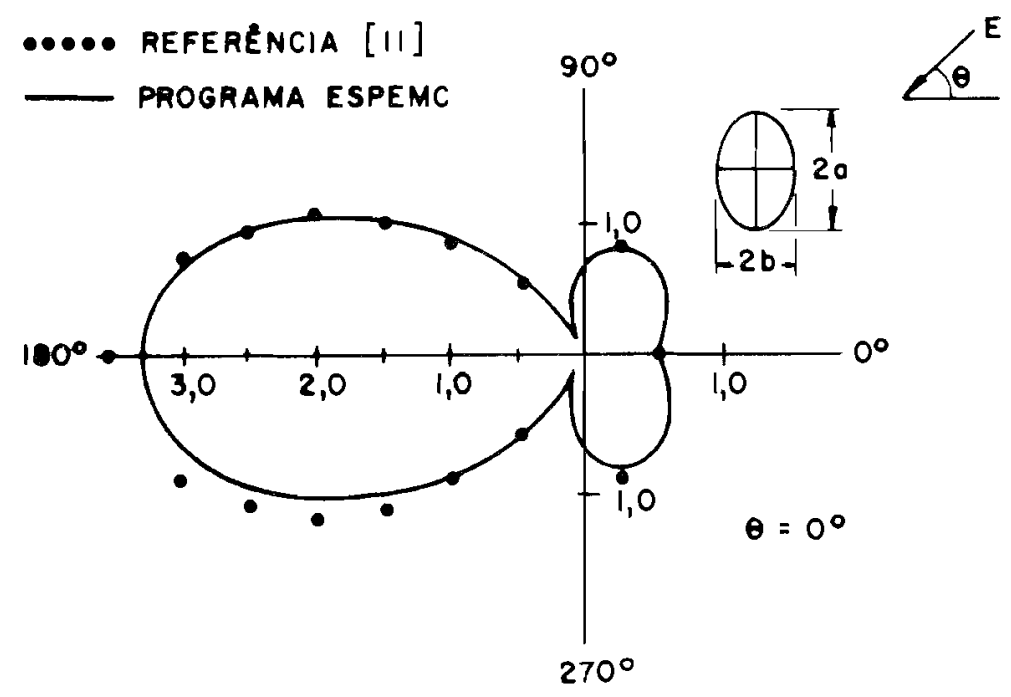

Figura 8. Magnitude do diagrama de espalhamento de um cilindro elítico homogêneo com $\epsilon_{r}=2, a=0,57 \lambda$ e $b=0,24 \lambda$.

O programa ESPEMC foi, entāo, usado no caso de um cilindro circular de raio $r_{c}=0,2 \lambda$ e com permissividade relativa dada por

$\epsilon_{\mathrm{r}}=\left[5-4\left(\mathrm{x} / \mathrm{r}_{\mathrm{c}}\right)^{2}\right] /\left[1+4\left(\mathrm{y} / \mathrm{r}_{\mathrm{c}}\right)^{2}\right]$

Na Fig. 10, estão mostradas as magnitudes dos diagramas de espalhamento obtidos a partir do programa e de [11], observando-se, entre estas curvas, diferenças menores que $10 \%$. Finalmente, as cascas cilíndricas de seção reta circular e semi-circular foram analisadas, computando-se suas larguras de eco, $W(\phi)$. Estas curvas estão mostradas, juntamente com os gráficos de [26], nas figuras $11 \mathrm{e} 12$. As diferenças observadas entre os resultados do programa ESPEMC e os de [26] são menores do que $15 \%$.

Para se estudar a relação entre a precisão dos resultados e a densidade de elementos na malha (número médio de intervalos radiais por comprimento de onda), foram efetuados testes com cilindros circulares de diferentes raios 


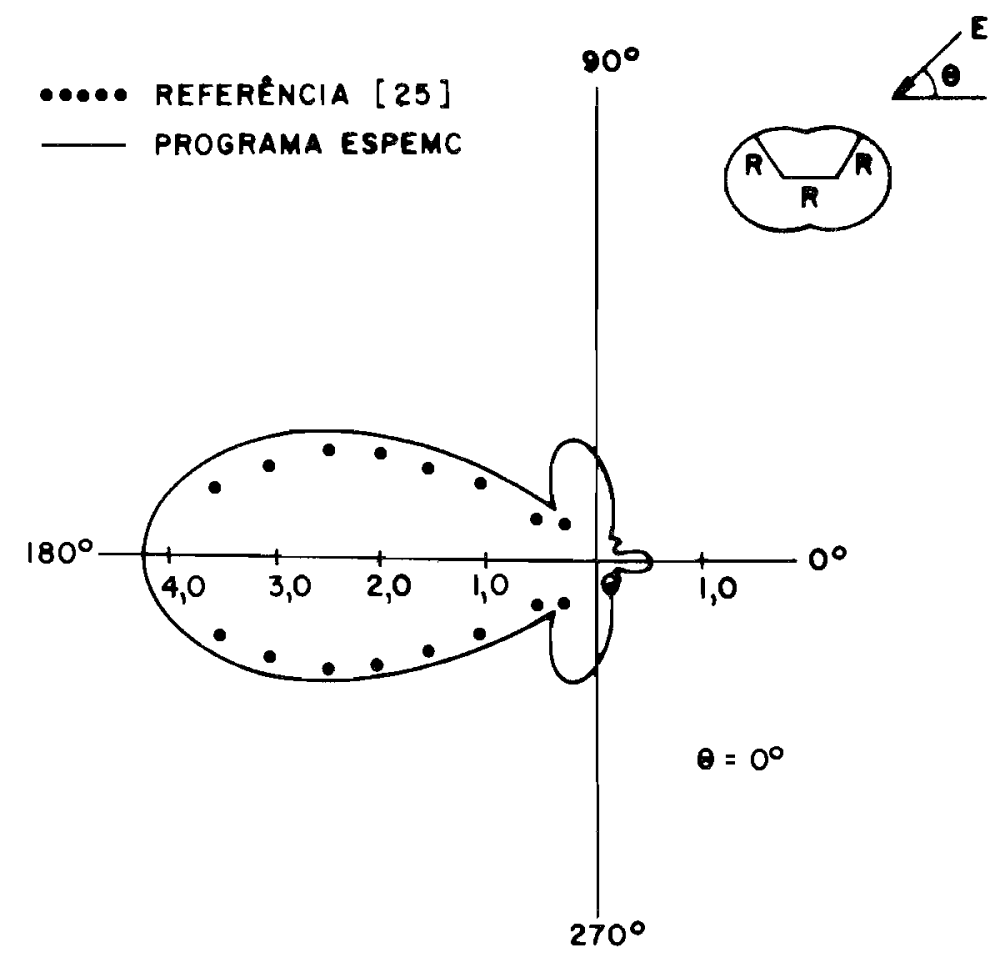

Figura 9. Magnitude do diagrama de espalhamento de dois cilindros homogêneos superpostos com $\epsilon_{\mathrm{r}}=4$ e $\mathrm{R}=0,3 \lambda$.

$\mathrm{r}_{\mathrm{C}}$ e variando o tamanho das malhas. Buscou-se, com isso, investigar como o erro $\left\{g(\phi) \mid\right.$ varia com relação ao parâmetro $k h=\epsilon_{r}^{1 / 2}\left(k_{0} h\right)$, onde $h$ é a medida do maior lado do elemento médio da malha. A Fig. 13 apresenta gráficos deste erro obtidos pelo programa ESPEMC e em [11], no caso de $r_{c}=0,4 \lambda$ e $\epsilon_{r}=4$. Uma comparação dos resultados mostra a eficiência superior da presente implementação, com os mesmos niveis de precisão sendo alcançados para valores menores de kh, isto é, com malhas menos densas.

Dentre as qualidades do método de unimomento freqüentemente apontadas, foram comprovadas por este trabalho as seguintes:

i) a introdução de um contorno circular artificial permite resolver separadamente os problemas interior e exterior, possibilitando um tratamento distinto para a região inomogênea e/ou de geometria complexa; 


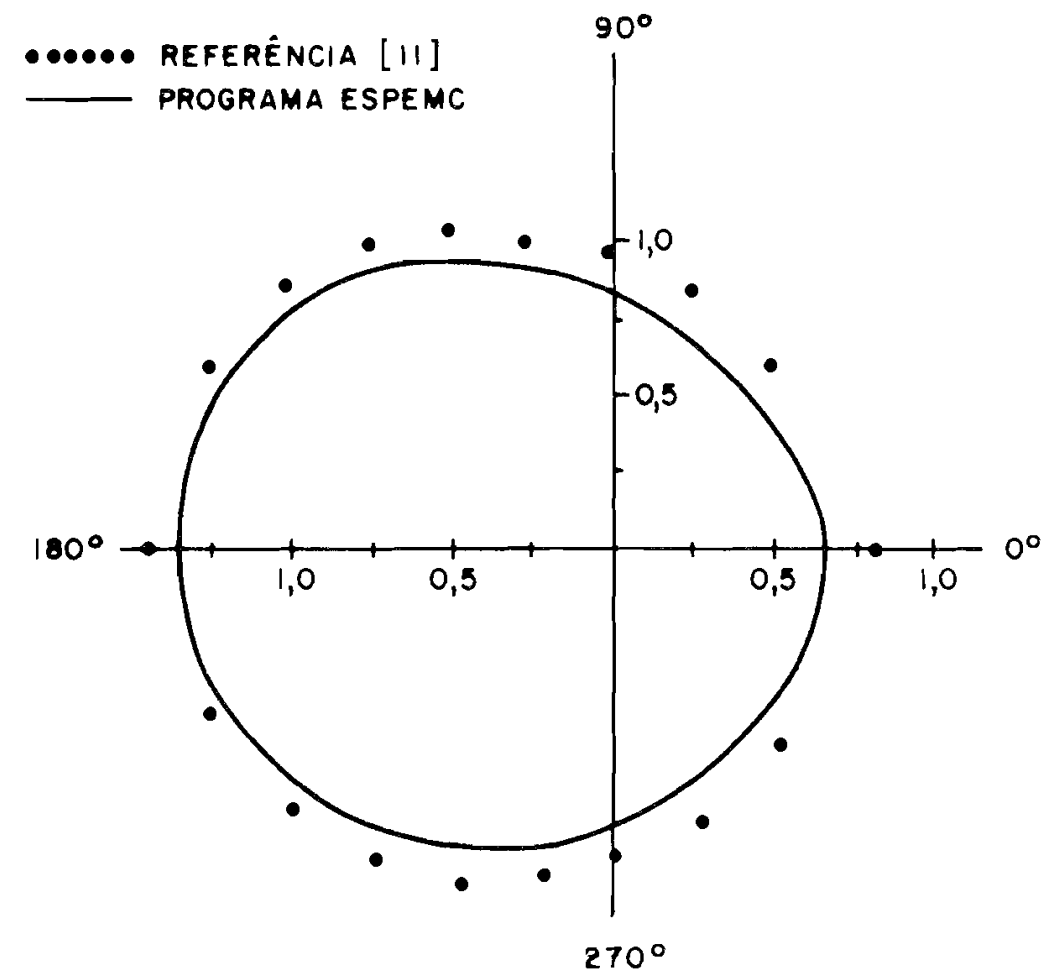

Figura 10. Amplitude do diagrama de espalhamento de um cilindro circular não-homogêneo com $r_{C}=0,2 \lambda_{\text {e }}$ $\epsilon_{\mathrm{r}}=\left[5-4\left(x / \mathrm{r}_{\mathrm{c}}\right)^{2}\right] /\left[1+4\left(\mathrm{y} / \mathrm{r}_{\mathrm{c}}\right)^{2}\right],\left(\theta=0^{\circ}\right)$.

ii) como as expansōes interior e exterior são acopladas sobre um círculo, basta calcular uma vez os valores das funçōes de Hankel e de suas derivadas;

iii) grande parte do esforço computacional independe do campo incidente, tornando muito econômico o cálculo simultâneo do espalhamento para várias ondas incidentes.

A principal característica da implementação aqui apresentada é o uso da malha anular conforme, que provou ser versátil com relação aos problemas abordados. Mesmo envolvendo o projeto de um gerador automático de malhas, ela ainda é computacionalmente simples se comparada aos métodos que utilizam elementos inomogêneos. 


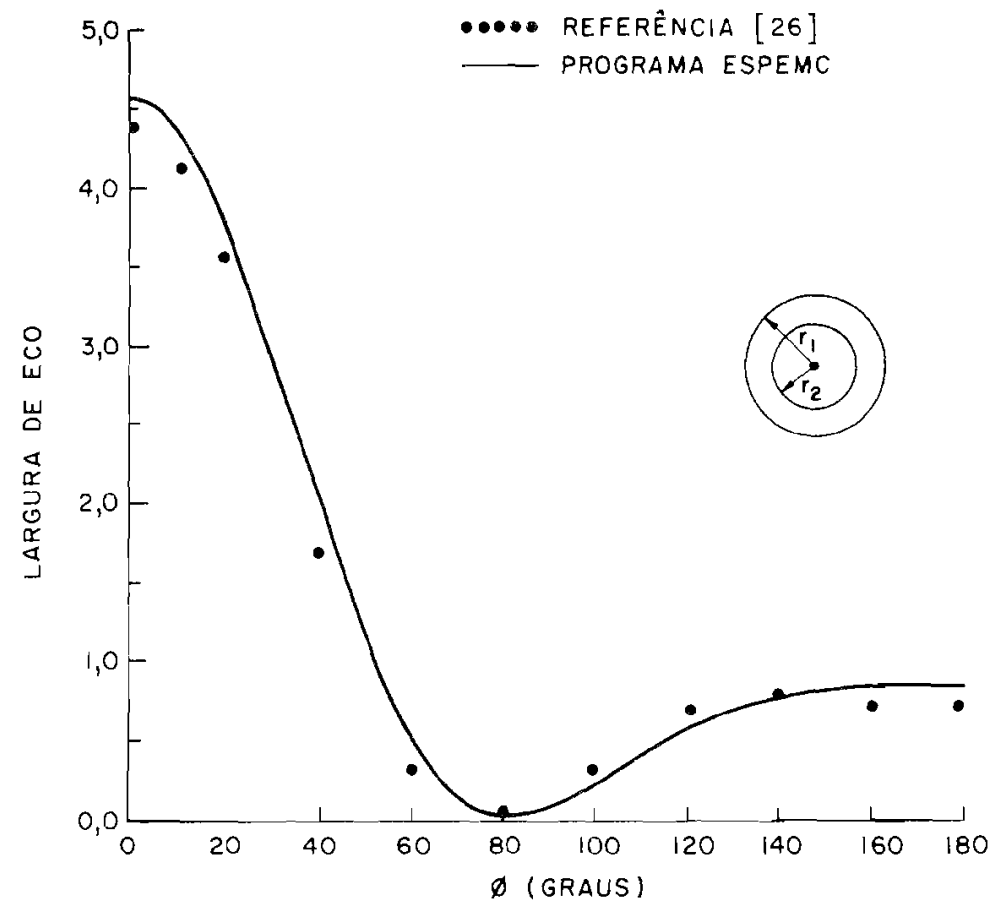

Figura 11. Largura de eco de uma casca cilíndrica circular com $\epsilon_{r}=4, r_{1}=0,3 \lambda$ e $r_{2}=0,25 \lambda\left(\theta=0^{\circ}\right)$.

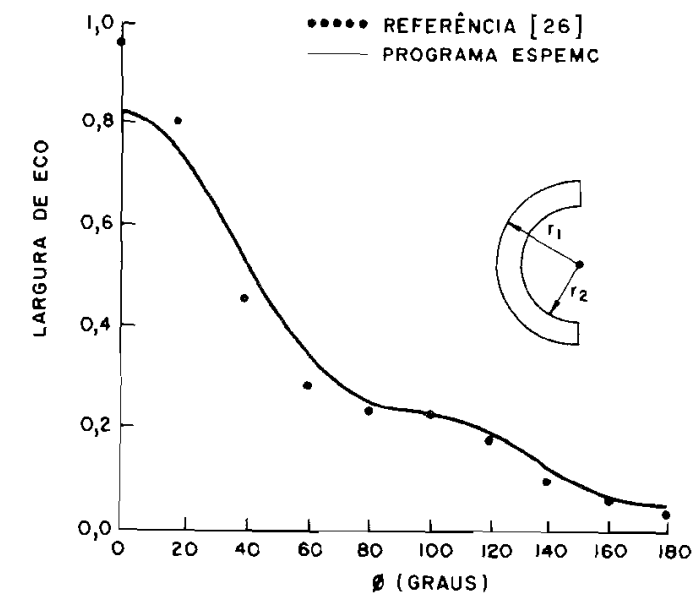

Figura 12. Largura de eco de uma casca cilíndrica semi-circular com $\epsilon_{\mathrm{r}}=4, r_{1}=0,3 \lambda$ e $r_{2}=0,25 \lambda\left(\theta=0^{\circ}\right)$. 
Esforços estão, agora, em andamento no sentido de estender a metodologia de modo a torná-la aplicável aos cálcuios de espalhamento por corpos tridimensionais de revolução.

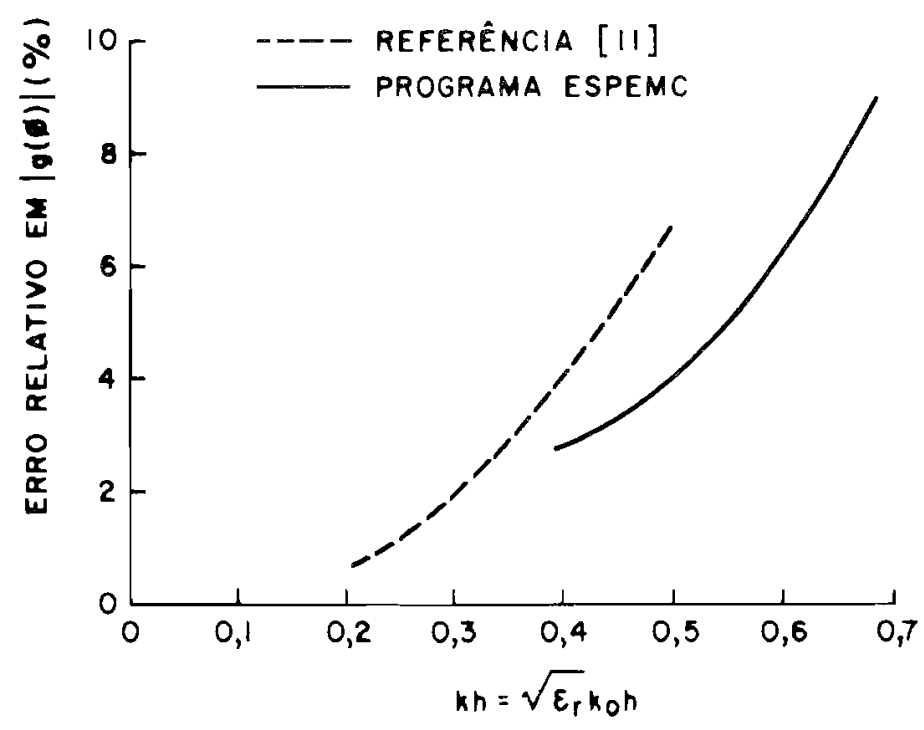

Figura 13. Erro relativo na amplitude de $g(\phi)$ em função do parâmetro da malha kh.

\section{Referências}

[1] K. K. Mei, "On the Integral Equations of Thin Wire Antennas", IEEE Transactions on Antennas and Propagation, vol. AP-13, n. 3 , Maio 1965, pp. 374-378.

[2] A. J. Poggio e E. K. Miller, "Integral Equation Solutions of Three-Dimensional Scattering Problems", em "Computer Techniques for Electromagnetics", R. Mittra (editor), Pergamon Press, Oxford, 1973.

[3] S. H. Lu e K. K. Mei, "Cylindrical Antennas in Uniaxial Media", Alta Frequenza, vol. 41, n.o 4, Junho 1972, pp. 290-298.

[4] C. Y. Ting, "Infinite Cylindrical Dielectric Coated Antennas", Radio Science, vol. 2, n. 3, Março 1967, pp. 325-335.

[5] P. Silvester e M. S. Hsieh, "Finite-Element Solution of 2-Dimensional Exterior-Field Problems", Proceedings of the IEE, vol. 118, Dezembro 1971, pp. 1743-1747. 
[6] B.H. McDonald e A. Wexler, "Finite-Element Solution of Unbounded Field Problems", IEEE Transactions on Microwave Theory and Techniques, vol. MTT-20, n. 12 , Dezembro 1972, pp. 841-847.

[7] C. A. Brebbia e S. Walker, "Boundary Element Techniques in Engineering", Newnes-Butterworths, London, 1980.

[8] S. P. Marin, "Computing Scattering Amplitudes for Arbitrary Cylinders", IEEE Transactions on Antennas and Propagation, vol. AP-30, n.' 6, Novembro 1982, pp. 1045-1049.

[9] O. C. Zienkiewicz, W. D. Kelly e P. Bettess, "The Coupling of the Finite Element Method and Boundary Solution Procedures", International Journal on Numerical Methods in Engineering, vol. 11, 1977, pp. 355-375.

[10] K. K. Mei, "Unimoment Method of Solving of Antenna and Scattering Problems", IEEE Transactions on Antennas andPropagation, vol. AP-22, n. 6, Novembro 1974, pp 760-766.

[11] S. K. Chang e K. K. Mei, "Application of the Unimoment Method to Electromagnetic Scattering of Dielectric Cylinders", IEEE Transactions on Antennas and Propagation, vol. AP-24, n. 1 , Janeiro 1976, pp. 35-42.

[12] M. A. Morgan e K. K. Mei, "Finite Element Computation of Sacattering by Inhomogeneous Penetrable Bodies of Revolution", IEEE Transactions on Antennas and Propagation, vol. AP-27, n.. 2, Março 1979, pp. 202-214.

[13] M. A. Morgan, C. H. Chang, S. C. Hill e P. W. Barber, "Finite ElementBoundary Integral Formulation for Electromagnetic Scattering", Journal of Wave Motion, vol.6, Janeiro 1984, pp. 91-103.

[14] R. F. Harrington, "Time-Harmonic Electromagnetic Fields", McGraw-Hill, New York, 1961.

[15] P. M. Berg e J.T. Fokkema, "The Rayleigh Hypothesis in the Theory of Diffraction by a Cylindrical Obstacle", IEEE Transactions on Antennas and Propagation, vol. AP-27, n. 5 , Setembro 1979, pp. 577-583.

[16] D. R. Wilton e R. Mittra, "A New Numerical Approach to the Calculation of Electromagnetic Scattering Properties of Two-Dimensional Bodies of Arbitrary Cross Section", IEEE Transactions on Antennas and Popagation, vol. AP-20, n. ${ }^{\circ}$, Maio 1972, pp. 310-317.

[17] C. R. Mullin, R. Sandburg e C. O. Velline, "A Numerical Technique for the Determination of Scattering Cross Sections of Cylinders of Arbitrary 
Geometrical Cross Section", IEEE Transactions on Antennas and Propagation, vol. AP-13, n. 1, Janeiro 1965, pp. 141-149.

[18] I. Stackgold, "Boundary Value Problems of Mathematical Physics", MacMillan, London, 1967, vol. 2, Capítulo 8.

[19] G. Strang e G. E. Fix, "An Analysis of the Finite Element Method", Prentice-Hall, Englewood Cliffs, New Jersey, 1973, capítulos 1 e 2.

[20] M. B. F. Chaves, "Espalhamento Eletromagnético por Cilindros Dielétricos Inomogêneos", Dissertação de Mestrado, Departamento de Engenharia Elétrica, PUC/Rio, Dezembro 1986.

[21] I. Holand e K. Bell, "Finite Element Methods in Stress Analysis", Tapir, Trendheim, Norway, 1969.

[22] A. C. Galeão, J. N. Guerreiro e H. J. Barbosa, "Um Sistema Automático para Análise Estrutural de Tubulações", Anais do 1. Congresso Latino Americano sobre Métodos Computacionais para Engenharia, Porto Alegre, Dezembro 1979, vol. 1, pp. 109-128.

[23] K. J. Bathe e I. T. Wilson, "Numerical Methods in Finite Element Analysis", Prentice-Hall, Inc., New Jersey, 1976.

[24] "IMSL Library Reference Manual", IMSL, Inc., Houston, 1984.

[25]K. K. Mei, M. Morgan e S. K. Chang, "Finite Methods in Electromagnetic Scattering", em "Electromagnetic Scattering",P.L.Uslenghi (editor), Academic Press, Inc., New York, 1978.

[26] J. H. Richmond, "Scattering by Dielectric Cylinder of Arbitrary CrossSection Shape", IEEE Transactions on Anternas and Propagation, vol. AP-13, n. 3, Maio 1965, pp. 334-341.

CARLOS GUSTAVO S. MIGLIORA nasceu no Rio de Janeiro em 18 de abril de 1952. Formou-se em Engenharia Elétrica - Telecomunicações na PUC/Rio, em 1974, e obteve o grau de Mestre em Ciências em Engenharia Elétrica, na mesma Universidade, em 1976. Concluiu o doutoramento no Polytechric Institute of New York, em 1980, com o título de PhD em Eletrofísica. Desde então leciona na PLC/Rio e trabalha em pesquisas no Centro de Estudos em Telecomunicações da Universidade Católica (CETUC). Suas áreas de interesse são o eletromagnetismo aplicado e a propagação de ondas eletromagnéticas em meios naturais. 
MIRIAM B. FERNANDES CHAVES nasceu em Penedo, Alagoas, em 3 de novembro de 1961. Concluiu o curso de Engenharia Elétrica na Universidade Santa Ursula, em 1983, e obteve o grau de Mestre em Ciências em Engenharia Elétrica, na PUC/Rio, em 1986. Desde 1983 é Assistente de Pesquisa no Laboratório Nacional de Computação Científica do CNPq, onde realiza pesquisas na área de métodos numéricos aplicados à propagação eletromagnética. Atualmente está cursando o doutoramento em Engenharia Elétrica na PUC/Rio. 